\title{
Analysis of a Tip Correction Factor for Horizontal Axis Turbines
}

\author{
A. Wimshurst and R. H. J. Willden \\ Department of Engineering Science, University of Oxford, Parks Road, Oxford, OX1 3PJ, UK.
}

\begin{abstract}
It is imperative to include three-dimensional tip flow corrections when using low order rotor models that rely on the flow independence principle to compute the blade forces. These corrections aim to account for the effect of pressure equalisation at the tips and the accompanying spanwise pressure gradients on the outboard sections, by reducing the computed axial and tangential forces as the blade tips are approached. Whilst Glauert type corrections are conventionally employed for actuator disc type computations, alternative corrections are required for actuator line computations as they use a finite blade representation. We present actuator line computations of the Model Rotor Experiments in Controlled Conditions (MEXICO) rotor to investigate tip corrections. Using the tip correction factor proposed by Shen et al. (Wind Energy 2005; 8:457-475), the actuator line computations show an improvement in accuracy over similar computations undertaken without a tip correction factor included. Further improvement to the blade loading is achieved by re-calibrating the tip correction factor using data extracted from blade resolved computations of the MEXICO rotor. From the rotor resolved computations, the tip loss (reduction in the blade loading on the outboard sections) is found to be more aggressive in the tangential direction than the axial direction. To account for this, we re-calibrate the tip correction factor separately in the axial and tangential diretions to develop new directionally dependent tip corrections. The resulting actuator line computations show a further improvement in accuracy of the tangential blade loading, resulting in better prediction of the rotor power.
\end{abstract}

Copyright (C) 2016 John Wiley \& Sons, Ltd.

\section{KEYWORDS}

Tip Correction Factor, Tip Loss, MEXICO, Actuator Line Method, Rotor Aerodynamics, CFD

Correspondence

Department of Engineering Science, University of Oxford, Parks Road, Oxford, OX1 3PJ, UK

E-mail: Aidan.Wimshurst@eng.ox.ac.uk

Received...

\section{INTRODUCTION}

At the tip of a 3D rotor blade, the pressure must equalise between the pressure and suction surfaces. Hence, a spanwise pressure gradient develops near the blade tip, driving fluid from the pressure surface around the tip to the suction surface, leading to a radial flow component along the blade. This end flow component gives rise to the tip vortex that is shed from the blade. In addition, the pressure equalisation between the pressure and suction surfaces at the blade tip leads to a reduction in the suction peak on the blade suction surface and a reduction in the pressure peak on the blade pressure surface. The resulting spanwise pressure gradient progresses back along the blade from the blade tip, reducing the blade loading on the outboard sections (the region from $\sim 75 \%$ to $100 \%$ of the span). When comparing the blade sectional performance with that of 2D aerofoil sections, the axial and tangential forces per unit span on these outboard sections reduce, leading to a reduction in integrated rotor thrust and torque.

Low order rotor models that operate using the flow independence principle, assume that each aerofoil section operates under locally 2D flow conditions (in the plane of the aerofoil section), neglecting the aerodynamic interaction between adjacent radial stations and spanwise flow effects. As a result, the spanwise pressure gradient on the outboard sections is neglected and the integrated rotor thrust and torque are often over-predicted. Hence, a correction is required for these 
methods to account for the lack of pressure equalisation at the blade tip that results in the over-prediction of the axial and tangential forces per unit span on the outboard sections. It should be noted that this type of correction is not the same as a Prandtl [1] or Goldstein [2] correction, which are applied in the blade element momentum (BEM) method [3] to account for the lack of discrete blade effects in the azimuthal averaging process (using Glauert's formulation [4]). In all methods that do not consider discrete blade effects, such as analytic BEM and Reynolds-averaged Navier-Stokes embedded blade element (RANS-BE) methods, both a pressure equalisation type correction and a Glauert [4] type correction are required [5]. However in this investigation, only the pressure equalisation type correction will be considered and will hereafter be referred to as a tip correction in this work.

Two different approaches have historically been adopted to correct reduced order methods that operate using the flow independence principle. The first approach corrects the lift and drag polars on the outboard sections, following observations from 3D rotor computations that the lift coefficient decreases and the drag coefficient increases on the outboard sections $[6,7,8]$. The second approach adopts 2D lift and drag polars along the entire span but corrects the resulting blade forces before they are applied to the flow field. While both methods aim to account for the same effect, they are distinctly different. In this investigation, the method of correcting the blade forces will be considered, as the method of correcting the lift and drag polars has recently been addressed by Wimshurst and Willden [8]. Rather than propose a new tip correction factor, the tip correction factor of Shen et al. [5] will be adopted, with the aim of assessing its applicability and improving its calibration based on new computational data that will be presented in this article.

This article begins by introducing the tip correction factor of Shen et al. [5], followed by the actuator line and blade resolved computations that will be used to calibrate it. The actuator line method was chosen as the low order rotor model in this investigation. For this type of model, a Glauert [4] type correction factor is not appropriate since the model already has a finite number of blades. After the actuator line and blade resolved computations have been performed, the calibration procedure will be described and new empirical coefficients for the correction factor will be derived. Finally, the calibrated correction factor will be applied to actuator line computations of the same rotor, demonstrating improved agreement on the outboard sections and subsequently the rotor thrust and power coefficients.

\section{TIP CORRECTION FACTOR}

To correct the blade forces for the effect of pressure equalisation, the axial and tangential forces per unit span $\left(F_{\text {ax }}\right.$ and $F_{\text {ta }}$ ) are multiplied by a correction factor $F_{1}$ before applying the forces to the flow field. The axial and tangential forces per unit span refer to the components of the blade force vector resolved in the streamwise direction and in the direction of blade rotation, rather than aligned parallel and perpendicular with the local aerofoil chord or the onset flow direction. In effect, they represent the thrust producing force and the torque producing force acting on the rotor.

Tip correction factors have been proposed by Lindenburg [9], Shen et al. [5] and Sant [10]. Of these, the tip correction factor of Shen et al. has been the most widely adopted in the literature and is therefore used in this investigation. It was decided not to propose a new correction factor at this stage, as the relative accuracy of the existing correction factors has not yet been comprehensively addressed and therefore the justification for a new correction factor was deemed to be insufficient. The tip correction factor $F_{1}$ proposed by Shen et al. [5] takes the following form,

$$
F_{1}=\frac{2}{\pi} \cos ^{-1}\left[\exp \left(-g_{1} \frac{N(R-r)}{2 r \sin \phi}\right)\right]
$$

$N$ represents the number of blades, $\phi$ the angle of the incident flow relative to the rotor plane (the sum of the angle of attack and the local pitch angle), $r$ the spanwise distance along the blade from the axis of rotation and $R$ the rotor radius. The functional form of $F_{1}$ was originally chosen to obtain the desired asymptotic behaviour at the blade $\operatorname{root}\left(F_{1} \rightarrow 1\right.$ as $r / R \rightarrow 0)$ and tip $\left(F_{1} \rightarrow 0\right.$ as $\left.r / R \rightarrow 1\right)$. In addition, for an infinitely bladed rotor $(N \rightarrow \infty)$ or a rotor running at an infinite tip speed ratio $(\sin (\phi) \rightarrow 0), F_{1} \rightarrow 1$ so that the rotor behaviour tends towards that of an actuator disc. Hence, the functional form of $F_{1}$ closely resembles that of the Glauert [4] correction factor $F$ (as they have the same asymptotic behaviour), with the exception of the additional parameter $g_{1}$ which is calibrated to experimental data.

$$
g_{1}=\exp \left(-c_{1}\left(N \lambda-c_{2}\right)\right)+0.1
$$

$\lambda$ represents the tip speed ratio and $c_{1}$ and $c_{2}$ are empirical coefficients $(0.125$ and 21.0$)$ that were originally calibrated by Shen et al. [5] to experimental data from the NREL Phase VI rotor at a low tip speed ratio $(\lambda=3.79)$ and the Swedish WG 500 rotor at a high tip speed ratio $(\lambda=14.0)$. These two experimental rotors were specifically chosen to cover a wide range of tip speed ratios, although Shen et al. note that more experimental and computational data would be required to improve the applicability of $g_{1}$ to other rotors.

In this investigation, new computational data for the Model Rotor Experiments in Controlled Conditions (MEXICO) rotor will be used to update the empirical coefficients $\left(c_{1}\right.$ and $\left.c_{2}\right)$, by providing additional data points for the intermediate 
tip speed ratios (6-10) between the NREL Phase VI and the Swedish WG 500 rotors. Given that the majority of full-scale wind turbines currently operate in the tip speed ratio range of 7-11, the updated empirical coefficients should improve the applicability of the tip correction factor to a wider range of rotors.

\section{MEXICO EXPERIMENTS}

The MEXICO rotor is a three bladed $4.5 \mathrm{~m}$ diameter horizontal axis wind turbine rotor, that was placed in the Large Lowspeed facility (LLF) of the German-Dutch Wind Tunnels (DNW) in December 2006. Each rotor blade was assembled from 3 different aerofoil sections. The DU91-W2-250 was used from 20-46\% of the span, the RIS $\varnothing$-A1-21 from 54$66 \%$ of the span and the NACA $64-418$ from $74-100 \%$ of the span [11]. Each of the sections was joined by proprietary transition pieces and the blade was blended into a cylinder at the root for attachment with the hub (approximately $0.54 \mathrm{~m}$ in diameter). For the blade resolved computations presented in this investigation, a computer aided design (CAD) file provided by the consortium that carried out the MEXICO experiments was used to ensure that the transition pieces were reproduced accurately. For the actuator line computations, the aerodynamic behaviour of the transition pieces was unknown. Therefore, the lift and drag polars were linearly interpolated from their (known) neighbouring aerofoil sections, following the approach of Shen et al. [12]. For the outboard sections (74\% to $100 \%$ of the span) the blade was entirely comprised of NACA 64-418 aerofoil sections and hence the transition regions were not expected to influence the tip losses and subsequent results of this investigation significantly.

In the LLF, the MEXICO rotor was placed in the $9.5 \mathrm{~m}$ high by $9.5 \mathrm{~m}$ wide open test section between the nozzle and collector. As a result, the blockage provided by the rotor was difficult to precisely define, as the open tunnel configuration allowed partial expansion of the nozzle streamtube before encountering the rotor. Previous investigations by Shen et al. [12] (which resolved the entire room that housed the LLF), concluded that blockage effects were insignificant and that an unblocked configuration could be assumed. Due to the significant computational expense of resolving the entire room, a virtually unblocked configuration ( $1 \%$ blockage ratio) was adopted for all the computations in this investigation.

The actuator line and blade resolved approaches adopted in this work were previously validated by Wimshurst and Willden $[13,8]$ using surface pressure and near wake particle image velocimetry (PIV) measurements from the MEXICO experiments. Despite the extensive available experimental data set, only axial flow conditions (zero yaw) at a fixed blade pitch angle of $2.3^{\circ}$ towards stall will be considered in this investigation, over a range of tip speed ratios from 6-10. These tip speed ratios were specifically chosen so that the rotor was operating before the onset of stall, as the appropriateness of the tip correction factor of Shen et al. [5] remains uncertain during stalled conditions.

\section{MEXICO ROTOR COMPUTATIONS}

\subsection{Actuator Line Computations}

The actuator line method of Sørensen and Shen [14] has been implemented in the open source code OpenFOAM (version 2.3.1) as a user defined shared object library. The potential flow equivalence method of Schluntz and Willden [15] was adopted to sample the flow field and the Gaussian smearing technique of Sørensen and Shen [14] was adopted to reapply the blade forces to the flow field. The width of the Gaussian distribution (controlled by the parameter $\epsilon$ ) was selected based on a previous investigation by Wimshurst and Willden [13], that gave the closest agreement with the experimental measurements. More specifically, a constant radial distribution of the smearing parameter along the blade span equal to half the average blade chord $(\epsilon=0.5 \times 0.1343 \mathrm{~m}=0.06715 \mathrm{~m})$, was found to give the best agreement.

The 2D lift and drag polars adopted in this investigation were computed by Wimshurst and Willden [8] using 2D Reynolds-averaged Navier-Stokes (RANS) computations of the DU91-W2-250, RIS Ø-A1-21 and NACA 64-418 aerofoil sections. These lift and drag polars were preferentially chosen over experimental lift and drag polars taken from wind tunnel experiments [11], as the aim of the actuator line computations was to reproduce the results from the blade resolved computations (the target solution), rather than the experimental measurements. For a comparison of the actuator line and blade resolved computations with the experimental measurements, the reader is referred to Wimshurst and Willden [8]. For the 2D aerofoil computations, the same steady flow solver, discretisation schemes and turbulence modelling adopted for the actuator line and blade resolved approaches was used and the computational mesh was constructed to closely resemble the mesh structure adopted for the 3D blade resolved computations. Fig. 1 shows the lift and drag polars that were computed for these aerofoil sections.

The computational domain for the actuator line simulations was taken as a cylinder with radius $10 R$ to ensure a low rotor blockage ratio (ratio of the rotor swept area to the cross-sectional area of the computational domain) of $1 \%$. A simplified nacelle was included at the centre of the cylindrical domain, with the rotor plane placed $5 R$ downstream from the domain inlet and $14 R$ upstream from the domain outlet. The nacelle geometry was based on the design drawings provided by 
(a)

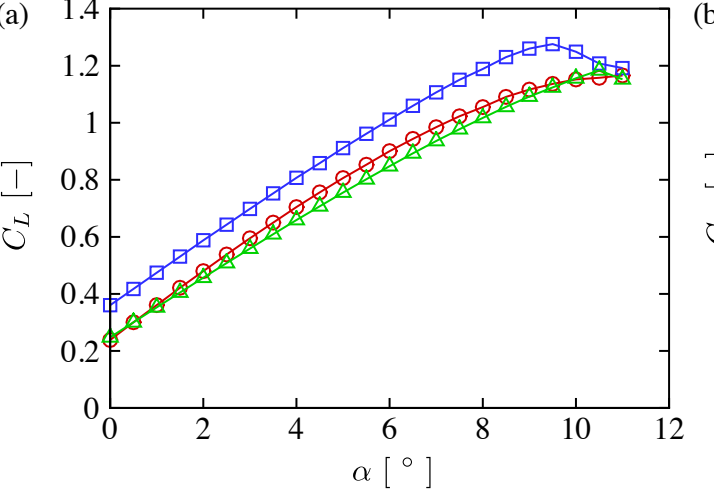

(b)

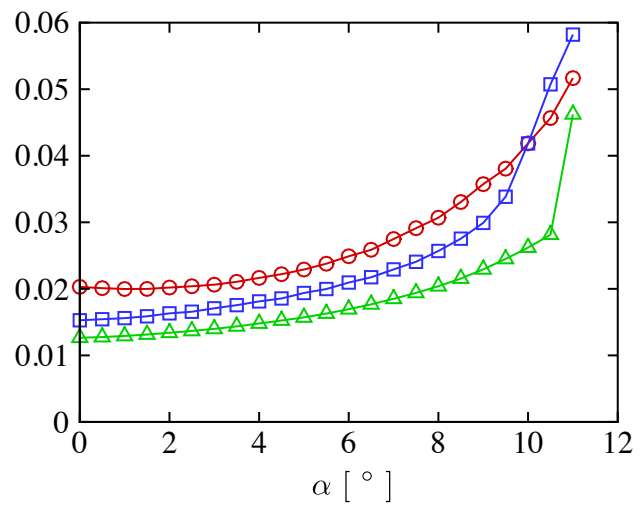

$\multimap$ DU91-W2-250 $\rightarrow$ RISOE-A1-21 $\triangle$ NACA 64-418

Figure 1. Sectional lift and drag coefficients computed for the DU91-W2-250, RIS $\varnothing-A 1-21$ and NACA 64-418 aerofoils at chord based Reynolds numbers of $0.5 \times 10^{6}, 0.6 \times 10^{6}$ and $0.7 \times 10^{6}$ respectively.

Boorsma and Schepers [16]. For consistency with the blade resolved computations, the tower geometry was not included in the computational domain. At the computational domain inlet, uniform profiles of velocity $U$, turbulent kinetic energy $k$ and specific dissipation rate $\omega$ were applied, based on the measured ambient turbulence intensity of $0.48 \%$ in the LLF and a length scale of $10 \%$ of the average blade chord [11]. At the computational domain outlet, zero gradient boundary conditions were applied for the velocity and turbulent scalars $(k$ and $\omega)$, while the static pressure was assigned a fixed value of zero. On the outer surface of the cylindrical domain, symmetry conditions were applied for all the flow variables.

The $k-\omega$ SST turbulence model was adopted for turbulence closure, with the SIMPLE algorithm of Patankar [17] for pressure-velocity coupling. Central differencing was applied for face interpolation of the Laplacian and gradient terms and the flux limited form of central differencing using the Sweby [18] limiter was applied for the convection terms. Temporal discretisation was achieved using second order backwards differencing and a fixed time step of $10^{-3} R / U_{\infty}$ was used for time advancement (an angular increment of $0.4^{\circ}$ per time step, at the design tip speed ratio).

A mesh sensitivity study was carried out and is reported in detail by Wimshurst and Willden [13]. Based on the axial and tangential forces per unit span, a polar mesh structure with 69 cells in the radial direction, 198 cells around the circumference and a streamwise cell dimension of $R / 30$ at the rotor plane, was found to be sufficient to compute the blade loading. This level of mesh resolution was consistent with that adopted by Shen et al. [12], who performed similar actuator line computations of the MEXICO rotor. Having identified an appropriate level of mesh refinement at the design tip speed ratio $(\lambda=6.67)$, additional actuator line computations were carried out at tip speed ratios of $6,7,8,9$ and 10 . These computations will be compared with the blade resolved computations in the next section.

\subsection{Blade Resolved Computations}

As the computed tip speed ratios $(6,7,8,9$ and 10) were specifically chosen before the onset of stall, the multiple reference frame (MRF) approach of Luo et al. [19] was adopted for simulating the rotor rotation, to significantly reduce the total compute time. Furthermore, as uniform inflow conditions were assumed and the tower was neglected, rotational symmetry could be utilised. More specifically, the computational domain was taken as a $120^{\circ}$ wedge (a third of a circular cylinder), with periodic boundary conditions applied on the sides of the domain, as shown in Fig. 2.

In the meshing process, the overall domain was first subdivided into separate inner and outer domains. The inner domain had length $0.7 R$, radius $1.25 R$ and contained a single blade, the nose cone and a section of the nacelle. The outer domain contained the domain boundaries and the remainder of the nacelle. Separate block structured meshes were created in the inner and outer domains and were coupled at the interface by an arbitrary mesh interface (AMI), using the face interpolation procedure of Farrell and Maddison [20] that is available in OpenFOAM. The separation of the computational domain into separate inner and outer domains was adopted so that a refined block structured mesh could be fitted around the blades in the inner domain, without having to maintain the structure of these blocks through to the outer domain (which would have resulted in a significant increase in total cell count).

A C-C type blocking topology was fitted around the blade in the inner domain, as shown in Fig. 3. The C-C type topology was adopted in order to retain the rotational symmetry at the sides of the $120^{\circ}$ wedge shaped domain and thus allow periodic boundary conditions to be applied. In addition, this blocking topology allowed independent control of the boundary layer cells in the wall normal direction, along the blade chord and along the blade span. 


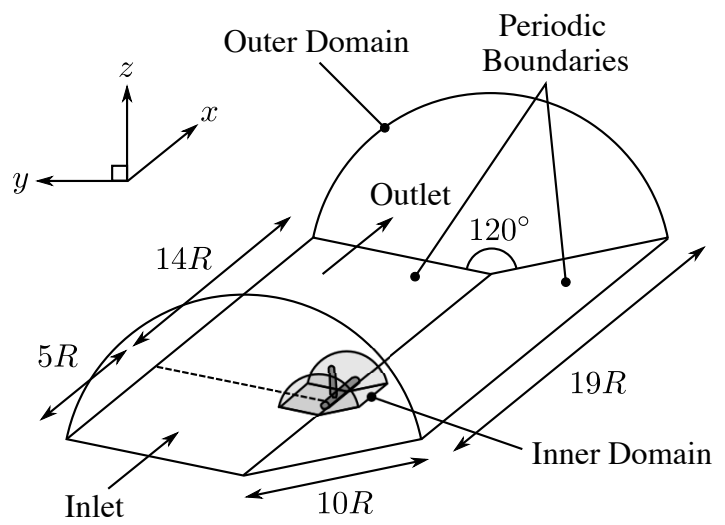

Figure 2. Diagram of the computational domain for the blade resolved computations. The streamwise flow direction is in the positive $x$ direction and the rotor rotates clockwise as viewed from the front.

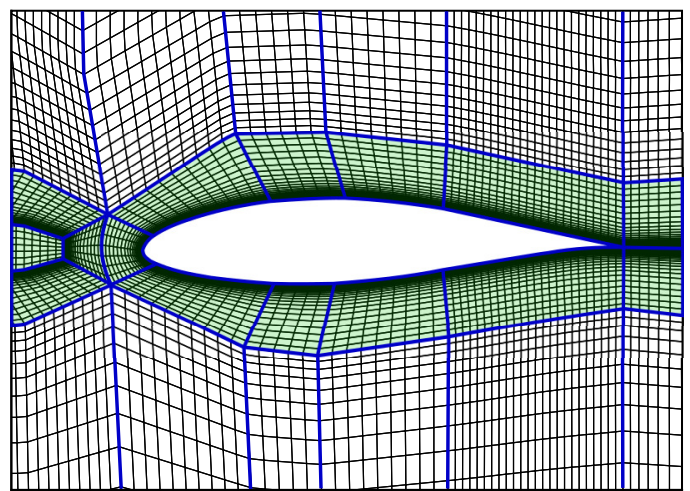

Figure 3. A slice through the coarse mesh normal to the blade axis at $r / R=0.8$. The blue lines indicate the blocking edges and the green shaded area highlights the C-C blocking topology.

A mesh sensitivity study was performed and is reported in detail by Wimshurst and Willden [8], to advise the distribution of cells in the chordwise direction, spanwise direction and in the wall normal direction. A summary of the mesh parameters adopted for this investigation is provided in Table I, including the chordwise spacing at the leading edge (LE), trailing edge (TE), the wall adjacent cell centroid height $\left(y_{0}\right)$, the number of chordwise cells along the pressure and suction surfaces individually $\left(N_{c}\right)$, the growth ratio normal to the wall $(G)$, the number of spanwise cells $\left(N_{s}\right)$, the spanwise cell dimension at the blade tip $\left(\Delta_{\text {tip }}\right)$, the number of cells on the blade pressure and suction surfaces individually $\left(N_{\text {surf }}\right)$ and the total number of cells in the inner domain $\left(N_{\text {cells }}\right)$. For all meshes, the spanwise cell dimension at the blade tip $\left(\Delta_{\text {tip }}\right)$ was held constant over the tip region (from $100-96 \%$ of the blade span) and then expanded with a linear growth ratio of 1.15 back along the span (from $96-80 \%$ of the blade span).

Table I. Summary of the parameters adopted for the blade resolved mesh sensitivity study of Wimshurst and Willden [8], expressed in terms of the chord length $c_{0.8 R} \approx 0.12 \mathrm{~m}$ at $r / R=0.8$. The wall normal distance $\left(y_{0}\right)$ corresponds with a maximum $y^{+}<5$ over the entire surface.

\begin{tabular}{cccccccccccc}
\hline Mesh & $\mathrm{LE} / c_{0.8 R}$ & $\mathrm{TE} / c_{0.8 R}$ & $y_{0} / c_{0.8 R}$ & $N_{c}$ & $G$ & $N_{s}$ & $\Delta_{\text {tip }} / c_{0.8 R}$ & $N_{\text {surf }}$ & $N_{\text {cells }}$ & $C_{T}$ & $C_{P}$ \\
\hline Coarse & $1.0 \mathrm{e}-2$ & $8.3 \mathrm{e}-3$ & $2.5 \mathrm{e}-4$ & 58 & 1.2 & 130 & $1.8 \mathrm{e}-2$ & 7,540 & $5,749,953$ & 0.7657 & 0.4338 \\
Medium & $1.0 \mathrm{e}-2$ & $4.2 \mathrm{e}-3$ & $2.5 \mathrm{e}-4$ & 78 & 1.1 & 188 & $1.3 \mathrm{e}-2$ & 14,664 & $9,792,087$ & 0.7775 & 0.4445 \\
Fine & $1.0 \mathrm{e}-2$ & $2.1 \mathrm{e}-3$ & $2.5 \mathrm{e}-4$ & 98 & 1.075 & 210 & $8.3 \mathrm{e}-3$ & 20,580 & $14,209,871$ & 0.7817 & 0.4455 \\
\hline
\end{tabular}

Based on the spanwise distribution of the axial and tangential forces per unit span at the design tip speed ratio $(\lambda=6.67)$, the medium mesh adopted by Wimshurst and Willden [8] was deemed to be sufficient for this investigation. More specifically, the axial force per unit span computed with the medium mesh was within $0.85 \%$ of that of the fine mesh at $r / R=0.8$ and the tangential force per unit span was within $1.2 \%$ of that of the fine mesh at $r / R=0.8$. In addition, 
the blade resolved computations gave good agreement with the experimental measurements presented by Schepers et al. [11] and with similar blade resolved computations performed by Bechmann et al. [21] (see Wimshurst and Willden [8] for the full comparison).

\subsection{Simulated Blade Loads}

Fig. 4 shows the axial and tangential forces per unit span $\left(F_{\mathrm{ax}}\right.$ and $\left.F_{\mathrm{ta}}\right)$ computed with the actuator line and blade resolved approaches. The actuator line computations over-predicted the axial and tangential forces per unit span on the outboard sections $(r / R>0.75)$, for all tip speed ratios. This is because, in its original formulation with no correction, the effect of pressure equalisation was not included in the actuator line approach and hence the axial and tangential forces per unit span were over-predicted. This over-prediction will be accounted for in the next section by multiplying the axial and tangential forces per unit span by the correction factor $\left(F_{1}\right)$ of Shen et al. [5]. Along the remainder of the span, the actuator line and blade resolved computations showed reasonable agreement.

\section{MAPPING THE CORRECTION FACTOR TO THE COMPUTATIONAL DATA}

In the following analysis, the actuator line results were used to represent the forces per unit span acting on the blade $\left(F_{\mathrm{ax}}^{\mathrm{AL}}\right.$ and $F_{\mathrm{ta}}^{\mathrm{AL}}$ ) with no pressure equalisation effects included, whereas the blade resolved results were used to represent the forces per unit span acting on the blade $\left(F_{\mathrm{ax}}^{\mathrm{BR}}\right.$ and $\left.F_{\mathrm{ta}}^{\mathrm{BR}}\right)$ with pressure equalisation effects included. Hence, the ratios of these two sets of forces $\left(F_{1 \text {,ax }}\right.$ and $\left.F_{1, \text { ta }}\right)$ represents the fractional reduction in axial and tangential forces per unit span on the outboard sections due to pressure equalisation at the blade tip, that was not included in the original actuator line method.

$$
\begin{aligned}
& F_{1, \mathrm{ax}}(r)=\frac{F_{\mathrm{ax}}^{\mathrm{AL}}(r)}{F_{\mathrm{ax}}^{\mathrm{BR}}(r)} \\
& F_{1, \mathrm{ta}}(r)=\frac{F_{\mathrm{ta}}^{\mathrm{AL}}(r)}{F_{\mathrm{ta}}^{\mathrm{BR}}(r)}
\end{aligned}
$$

Having computed the ratios of the axial and tangential forces per unit span $\left(F_{1, \text { ax }}\right.$ and $\left.F_{1, \text { ta }}\right)$ from the computational data, a function of the form given by $F_{1}$ (equation 1 ) was fitted to each of these ratios separately by using the spanwise distribution of $\phi$ (the sum of the angle of attack and local pitch angle) from the actuator line computations. The spanwise distribution of $\phi$ was taken from the actuator line computations rather than the blade resolved computations, as the angle of attack is not straightforward to accurately extract from blade resolved computations [8].

The best fit for the function $F_{1}$ was taken as that which minimised the sum of the squared residuals, through a least squares regression analysis. During the regression analysis, only the data on the outboard sections (between $r / R=0.80$ and 0.95 ) was used, as the tip correction factor was only intended to capture the effect of pressure equalisation on the outboard sections. The blade tip region $(r / R>0.95)$ was also excluded, as the data seemed to be unreliable in this region. A separate fit was carried out in the axial and tangential directions for each tip speed ratio and the best fit in each case yielded a unique value of $g_{1}$. The physical interpretation of $g_{1}$ is the strength of the tip correction factor at a given tip speed ratio. Its behaviour will be discussed in more detail in the next section.

Fig. 5 compares the computed ratios $F_{1 \text {,ax }}$ and $F_{1, \text { ta }}$ and the best fit of the function $F_{1}$ to the data in the axial and tangential directions. Most importantly Fig. 5 shows that the best fit to the computed data was considerably different in the axial and tangential directions. This is because the effect of pressure equalisation caused a greater reduction in tangential force per unit span than axial force per unit span. These observations were first made by Wimshurst and Willden [8] for the MEXICO rotor and indicate that a different degree of correction may be required in the axial and tangential directions. Such a correction can be readily achieved using the same fitting function $F_{1}$ but with different values of $g_{1}$ in the axial and tangential directions (here denoted $g_{1, \text { ax }}$ and $\left.g_{1, \text { ta }}\right)$. This in turn can be achieved with different empirical constants $\left(c_{1}\right.$ and $c_{2}$ ) in the axial and tangential directions, as will be shown in the next section.

The data fits in Fig. 5 were all partially restricted by the functional form of $F_{1}$. In some instances, the asymptotic behaviour that $F_{1} \rightarrow 0$ as $r / R \rightarrow 1$ prevented a more accurate fit on the outboard sections. For example, the sub-optimal fit in the tangential direction for the high tip speed ratio case $(\lambda=10)$, arose because the tangential force per unit span from the blade resolved computations tends to zero before the very tip. Such a situation can arise physically for $r / R<1$ as $L \sin (\phi)-D \cos (\phi) \rightarrow 0$ as $\phi \rightarrow 0$. This is more likely to occur at high tip speed ratios as flow angle relative to the rotor plane $(\phi)$ reduces with increasing tip speed ratio. These observations suggest that particularly at high tip speed ratios, an alternative form of $F_{1}$ may lead to a better fit, particularly if $F_{1}$ is not constrained to approach zero only at the very tip. However, proposing such a function is beyond the scope of this investigation. 

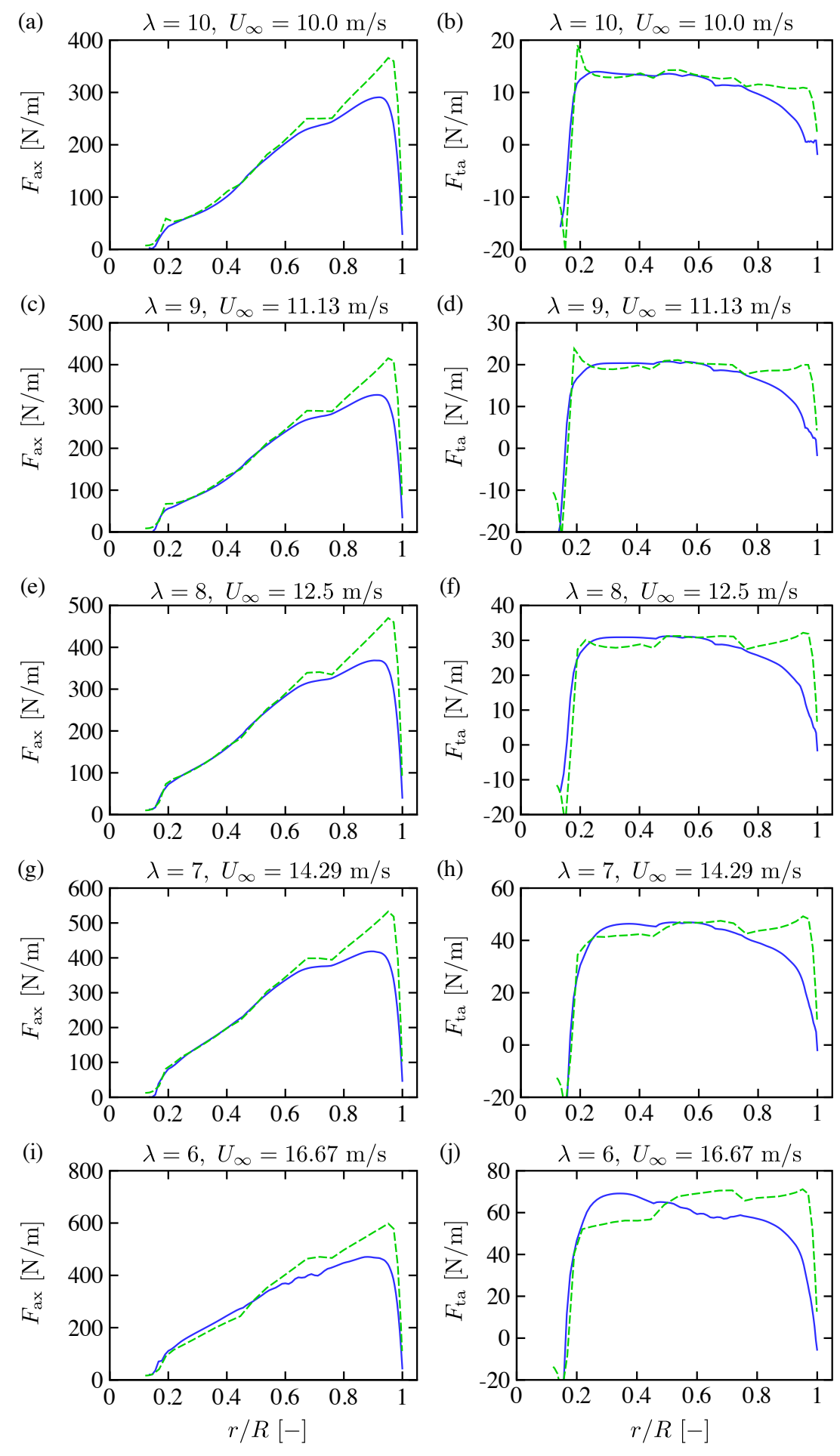

Actuator Line

Blade Resolved

Figure 4. Axial and tangential blade forces per unit span for tip speed ratios of 10, 9, 8, 7 and 6, using the actuator line and blade resolved approaches. 

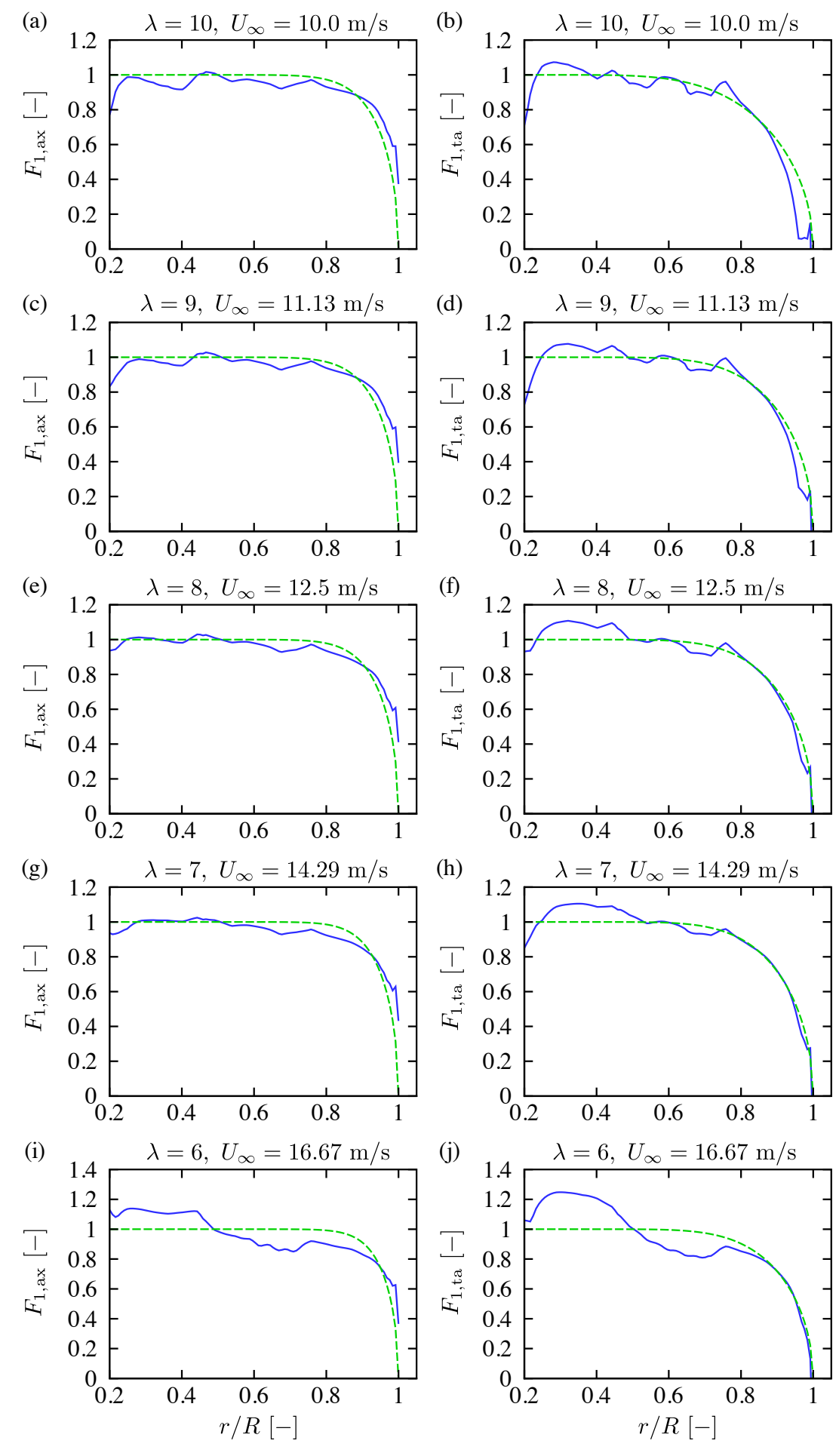

Computed Ratio

Best Fit

Figure 5. Curve fits of the computed data to the function $F_{1}$ in the axial and tangential directions, for tip speed ratios of 10, 9, 8, 7 and 6 .

\subsection{Computing new empirical coefficients}

To demonstrate the general behaviour of $F_{1}$, Fig. 6 (a) shows the variation of the tip correction factor $F_{1}$ with $g_{1}$ for a tip speed ratio of 7 (near the design operating condition for the MEXICO rotor). $g_{1}$ effectively controls the strength (or 
curvature) of the tip correction factor $F_{1}$, allowing it to better fit the computed data. Decreasing the value of $g_{1}$ decreases the value of $F_{1}$ and hence increases the correction applied to the axial and tangential forces per unit span. In effect, reducing $g_{1}$ leads to a stronger tip loss and hence a greater reduction in axial and tangential forces per unit span on the outboard sections.
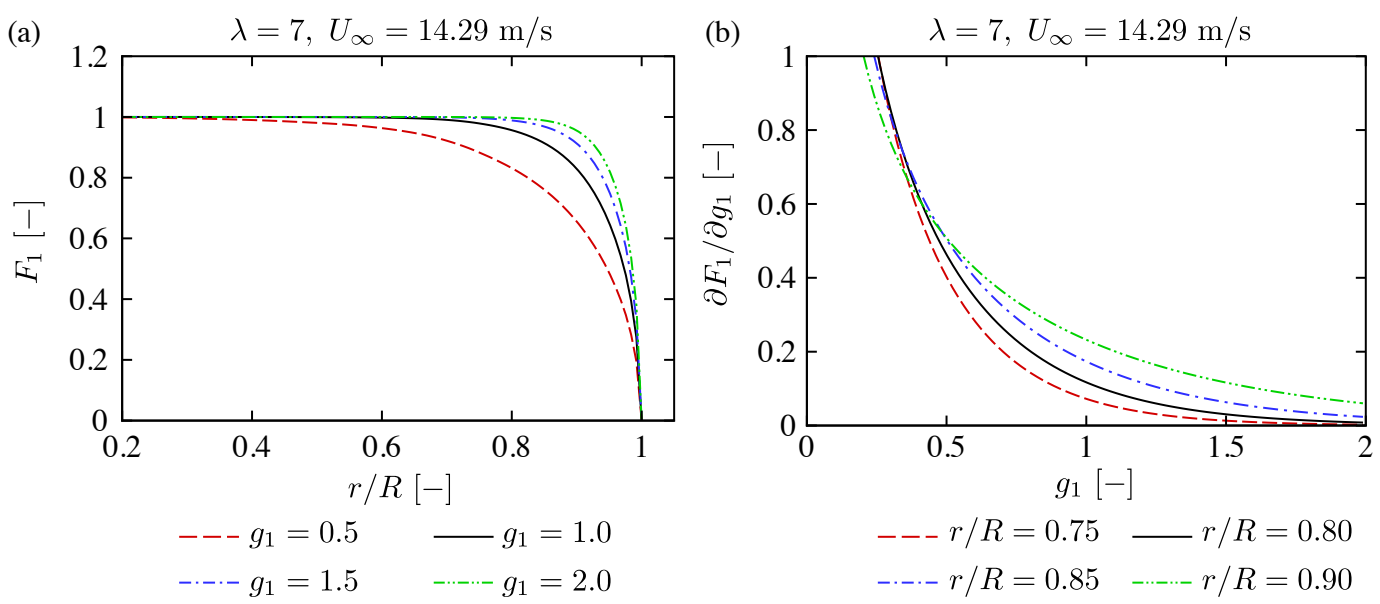

Figure 6. Variation of the tip correction factor $\left(F_{1}\right)$ of Shen et al. [5] and its derivative $\left(\partial F_{1} / \partial g_{1}\right)$ with $g_{1}$ and spanwise distance $r / R$.

Fig. 6 (b) shows the partial derivative $\partial F_{1} / \partial g_{1}$ as a function of $g_{1}$, on a few selected outboard sections of the blade $(r / R=0.75,0.8,0.85,0.9)$. The derivative can be interpreted as the sensitivity of $F_{1}$ to changes in $g_{1}$ (that may arise from an inaccurate curve fit). A large derivative implies that a small change in $g_{1}$ results in a large change in $F_{1}$ and hence a significant change in the axial and tangential forces per unit span. More specifically, a change of 0.1 in $F_{1}$ directly corresponds with a $10 \%$ change in the axial and tangential forces per unit span, which will have considerable consequences for the integrated rotor thrust and torque. Fig. 6 (b) shows that $F_{1}$ is more sensitive to changes in $g_{1}$ for low values of $g_{1}$. Low values of $g_{1}$ were found to occur at high tip speed ratios and hence $F_{1}$ is more sensitive to the curve fitting process at high tip speed ratios. Furthermore, the sensitivity of $F_{1}$ to changes in $g_{1}$ increases with $r / R$, further compounding the errors in outboard load reduction if $g_{1}$ is assessed inaccurately.

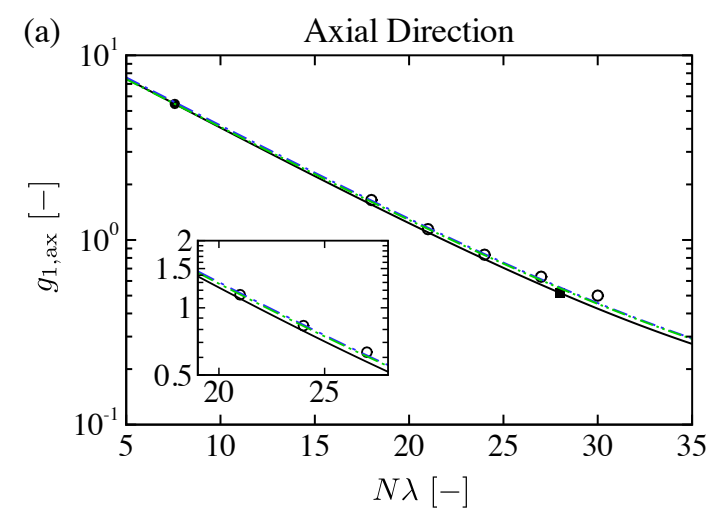

Shen et al. Correction (Original Coefficients) (b)

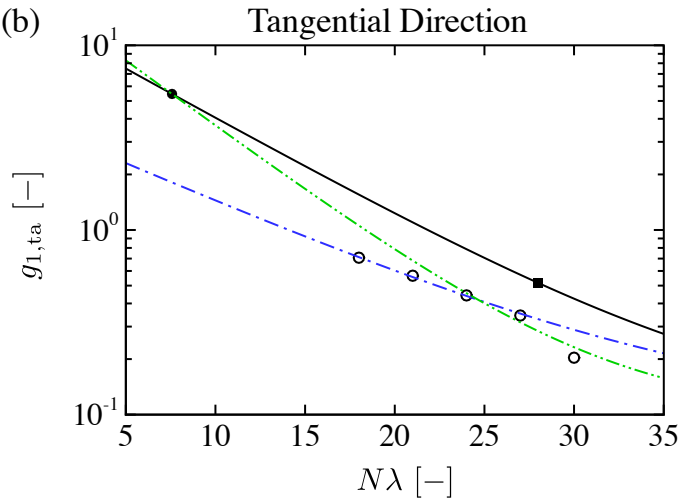

-.-.- Curve Fit $1 \quad-. .-.-$ Curve Fit 2

\section{- Computed Data Points \\ - NREL Phase VI (Shen et al.) \\ - Swedish WG 500 (Shen et al.)}

Figure 7. Computed values of $g_{1}$ in the (a) axial and (b) tangential directions. Curve fit 1 was performed using the computed data points for the MEXICO rotor alone (excluding the data point $N \lambda=30$ ). Curve fit 2 was performed using the computed data points for the MEXICO rotor (excluding the data point $N \lambda=30$ ), the NREL Phase VI rotor and the Swedish WG 500 rotor data points.

The computed values of $g_{1, \text { ax }}$ and $g_{1, \text { ta }}$ from the curve fitting process in section 5 are shown in Fig. 7. $g_{1, \text { ax }}$ and $g_{1, \text { ta }}$ have been plotted against the product of the tip speed ratio and number of blades $(N \lambda)$, rather than just the tip speed ratio $\lambda$, to allow direct comparison with equation 2. Data points for the NREL Phase VI rotor $(N=2, \lambda=3.79)$ and the 
Swedish WG 500 rotor $(N=2, \lambda=14.0)$ that were computed by Shen et al. [5], have also been included for reference. In addition to the curve fit of Shen et al. [5], two new curve fits have been included in Fig. 7. Curve fit 1 was carried out by fitting equation 2 to the derived MEXICO data points alone. The high tip speed ratio case $(N \lambda=30)$ was omitted from this curve fit, due to the previous difficulties in mapping the function $F_{1}$ to the computed data in section 1 , leading to an unreliable data point in Fig. 7. This curve fit represents the approach that might be taken to fit the correction factor to a specific rotor, for subsequent computations using the actuator line method. Curve fit 2 was carried out by fitting equation 2 to the MEXICO data points (excluding the high tip speed ratio data point at $N \lambda=30$ ), the NREL Phase VI rotor data point and the Swedish WG 500 rotor data point. This curve fit represents the approach that might be taken to move towards a more general correction factor that can be applied to new rotors, using all the available data.

In the axial direction, the original curve fit of Shen et al. [5] showed good agreement with the computed data points for the MEXICO rotor. This implied that the original correction factor would be a reasonable first approximation to correcting the axial force per unit span in the actuator line method. The new curve fits required slightly greater $g_{1, \text { ax }}$ than the original curve fit of Shen et al. [5] (approximately 0.05 or $4.3 \%$ greater at $N \lambda=21$ ), implying that a slightly greater $F_{1}$ (smaller load reduction) would have led to a better correction of the axial force per unit span. However, it can be deduced using a combination of Fig. 6 and Fig. 7 that these changes were likely to be small. For $N \lambda=21, g_{1, \text { ax }}=1.15$ (from Fig. 7) and the corresponding gradient $\partial F_{1} / \partial g_{1}=0.08$ at $r / R=0.8$ (from Fig 6). Hence a $4.3 \%$ change in $g_{1 \text {, ax }}$ would only result in approximately a $0.34 \%$ change in $F_{1}$ at $r / R=0.8$. Further substantive improvements to the tip correction factor in the axial direction would therefore require an alternative functional form for $F_{1}$, rather than improving the accuracy of the fitting process.

In the tangential direction, the original curve fit of Shen et al. [5] was considerably greater than the computed data points for the MEXICO rotor (approximately 0.53 or $96.1 \%$ greater at $N \lambda=21$ ). Therefore, the original curve fit was likely to considerably over-predict the value of $F_{1}$ required in the tangential direction and consequently over-predict the tangential force per unit span (and the rotor torque). For example, at $r / R=0.8$ and $N \lambda=21, g_{1, \text { ta }}=0.57$ and $\partial F_{1} \partial g_{1} \sim 0.38$. Hence an over-prediction of $96.1 \%$ in $g_{1, \text { ta }}$ would result in approximately a $36.5 \%$ over-prediction in $F_{1}$ and tangential force per unit span at $r / R=0.8$. Curve fit 1 , which was fitted to the MEXICO rotor data points alone, confirmed that an exponential fit (using equation 2) was also appropriate for $g_{1, \text { ta }}$ in the tangential direction. With lower values of $g_{1, \text { ta }}$, curve fit 1 was likely to produce lower values of $F_{1}$ in the tangential direction and hence lead to a greater reduction in tangential force per unit span than the original curve fit. Curve fit 2 did not appear to show good agreement with the computed data points for the MEXICO rotor. This is because the curve fit included the NREL Phase VI rotor at $N \lambda=7.58$, which lead to a significant distortion in the curve fit at high tip speed ratios. Until more experimental and computational data becomes available, this curve fit is likely to be unreliable due to the strong dependence on the single NREL Phase VI rotor data point.

Table II shows the empirical coefficients $c_{1}$ and $c_{2}$ that were computed from each of the exponential curve fits in Fig. 7. With reference to equation 2, $c_{1}$ represents the gradient of $g_{1}$ and $c_{2}$ represents the horizontal shift along the $N \lambda$ axis.

Table II. Empirical coefficients for the functions $g_{1, \text { ax }}$ and $g_{1, \text { ta. }}$.

\begin{tabular}{ccccc}
\hline & \multicolumn{2}{c}{$g_{1, \mathrm{ax}}$} & \multicolumn{2}{c}{$g_{1, \mathrm{ta}}$} \\
& $c_{1}$ & $c_{2}$ & $c_{1}$ & $c_{2}$ \\
\hline Shen et al. [5] & 0.125 & 21.0 & 0.125 & 21.0 \\
Curve Fit 1 & 0.1219 & 21.52 & 0.0984 & 13.026 \\
Curve Fit 2 & 0.1215 & 21.39 & 0.1652 & 17.732 \\
\hline
\end{tabular}

In the axial direction, $c_{1}$ and $c_{2}$ computed using curve fits 1 and 2 were within $2.8 \%$ and $2.4 \%$ of the values suggested by Shen et al. [5]. Furthermore, $c_{1}$ and $c_{2}$ were found to be slightly greater for curve fit 1 than curve fit 2 (corresponding with a steeper gradient and a shift to increased $N \lambda$ ), consistent with Fig. 7 . These observations further confirm that the values suggested by Shen et al. [5] can be used as a reasonable first approximation for actuator line computations of the MEXICO rotor. In the tangential direction $c_{1}$ and $c_{2}$ differed considerably between the curve fits, inferring that care should be taken when selecting the empirical coefficients in the tangential direction. Curve fit 1 (specific to the MEXICO rotor) had a $21.3 \%$ lower $c_{1}$ and a $38.0 \%$ lower $c_{2}$, corresponding with a shallower gradient and a shift to lower $N \lambda$. In contrast, curve fit 2 had a $32.2 \%$ higher $c_{1}$ and a $15.6 \%$ higher $c_{2}$, corresponding with a steeper gradient and shift to lower $N \lambda$. These values for $c_{1}$ and $c_{2}$ can be used as a reference as additional experimental and computational data becomes available for different rotors. In the next section, actuator line computations of the MEXICO rotor will be used to demonstrate the improved prediction of the axial and tangential forces per unit span that can be achieved by adopting the new empirical coefficients proposed in Table II. 


\section{CORRECTED ACTUATOR LINE COMPUTATIONS}

Fig. 8 shows the axial and tangential forces per unit span computed using the actuator line method and the tip correction factor $\left(F_{1}\right)$, with the empirical coefficients proposed by Shen et al. [5], the empirical coefficients from curve fit 1 and the empirical coefficients from curve fit 2 . In addition, the actuator line computations with no correction factor have been included in Fig. 8 for reference and the blade resolved computations have also been included to represent the target values.

Without a tip correction factor, the axial force per unit span was considerably over-predicted on the outboard sections. For example, at $r / R=0.9$ and $\lambda=7$ (near the peak operating point), the axial force per unit span was over-predicted by $17.8 \%$. With the tip correction factor included, the axial force per unit span was considerably reduced, leading to an over-prediction of only $4.3 \%$ (using the empirical coefficients of curve fit 1). As expected, the axial force per unit span using the empirical coefficients from the three different curve fits were all remarkably similar. More specifically, the maximum difference in the axial force per unit span between these three computations at $r / R=0.90$ was only $1.4 \%$. This observation confirms that the empirical coefficients of Shen et al. [5] would be a reasonable first approximation for actuator line computations of the MEXICO rotor, if the primary objective is the reproduction of the axial force per unit span. However, the axial force per unit span computed using these empirical coefficients still did not match the blade resolved computations completely. As discussed in section 5, this was due to the functional form of $F_{1}$, which did not always allow an optimal curve fit when fitted to the computational data. To address this discrepancy, alternative forms of $F_{1}$ could be adopted instead to give a closer agreement with the blade resolved computations. However, this is beyond the scope of the current investigation.

The tangential force per unit span was also considerably over-predicted without a tip correction factor applied. Due to the lower values of the tangential force per unit span than axial force per unit span, the percentage discrepancy between the actuator line and blade resolved computations was also much greater. For example, at $r / R=0.9$ and $\lambda=7$ the tangential force per unit span was over-predicted by $38.9 \%$ without a tip correction applied. The inclusion of the tip correction factor considerably reduced this discrepancy in all cases. However, the tangential force per unit span computed with the tip correction factor and empirical coefficients of Shen et al. [5], was still over-predicted for all the considered cases. For example, at $r / R=0.9$ and $\lambda=7$, the tangential force per unit span was over-predicted by $21.3 \%$. This is because $g_{1, \text { ta }}$ computed using the empirical coefficients of Shen et al. [5] was too large for the MEXICO rotor and hence $F_{1}$ was also too large, leading to an insufficient load reduction on the outboard sections.

Curve fits 1 and 2 lead to a further reduction in tangential force per unit span, improving the agreement with the blade resolved computations. At $r / R=0.9$ and $\lambda=7$ for example, the tangential force per unit span was only under-predicted by $1.3 \%$ using curve fit 1 and $3.9 \%$ by curve fit 2 . Overall, curve fit 1 gave the closest agreement with the blade resolved computations over all the considered tip speed ratios (with the exception of the high tip speed ratio case $N \lambda=30$ ), as this curve fit for $g_{1, \text { ta }}$ was carried out using only the MEXICO rotor data points. Nevertheless, curve fit 2 also showed a good agreement, although the tangential force per unit span was still over-predicted at the low tip speed ratio $(\lambda=6)$. This in turn was due to the over-prediction of $g_{1, \text { ta }}$ at low tip speed ratios, as the NREL Phase VI rotor was included in curve fit 2 for $g_{1, \mathrm{ta}}$.

Fig. 9 shows the rotor thrust and power coefficients $\left(C_{T}\right.$ and $C_{P}$ respectively), computed from the rotor thrust $(T)$ and power $(P)$ using equations 5 and 6 .

$$
\begin{aligned}
& C_{T}=\frac{T}{\frac{1}{2} \rho U_{\infty}^{2} \pi R^{2}} \\
& C_{P}=\frac{P}{\frac{1}{2} \rho U_{\infty}^{3} \pi R^{2}}
\end{aligned}
$$

Fig. 9 demonstrates the importance of accurate prediction of the axial and tangential forces per unit span on the rotor thrust and power coefficients. At a tip speed ratio of 7, the actuator line computations (with no correction) over-predicted $C_{T}$ by $0.07(8.7 \%)$ and $C_{P}$ by $0.067(15.1 \%)$. The inclusion of the tip correction factor reduced this discrepancy in all cases. For the thrust coefficient, all three actuator line computations using the tip correction factor showed close agreement with the blade resolved computations. This is because the spanwise distribution of the axial force per unit span showed close agreement between the cases, which in turn was due to the similar values of $g_{1 \text {, ax. }}$. For example at $\lambda=7$, the thrust coefficient was only over-predicted by a maximum of $0.011(1.4 \%)$ by these actuator line computations.

When adopting the tip correction factor with the original empirical coefficients of Shen et al. [5], the power coefficient reduced considerably in comparison with the actuator line computations that did not include a tip correction factor. At a tip speed ratio of 7 for example, the power coefficient was now only over-predicted by 0.028 (6.3\%). However, Fig. 8 shows that this was still sub-optimal as the tangential force per unit span was over-predicted on the outboard sections for all the considered tip speed ratios. Curve fits 1 and 2 both achieved better agreement with the blade resolved computations on the outboard sections and therefore experienced lower power coefficients than the actuator line computations with the original empirical coefficients of Shen et al. [5]. At a tip speed ratio of 7, the power coefficient was now only under-predicted by 

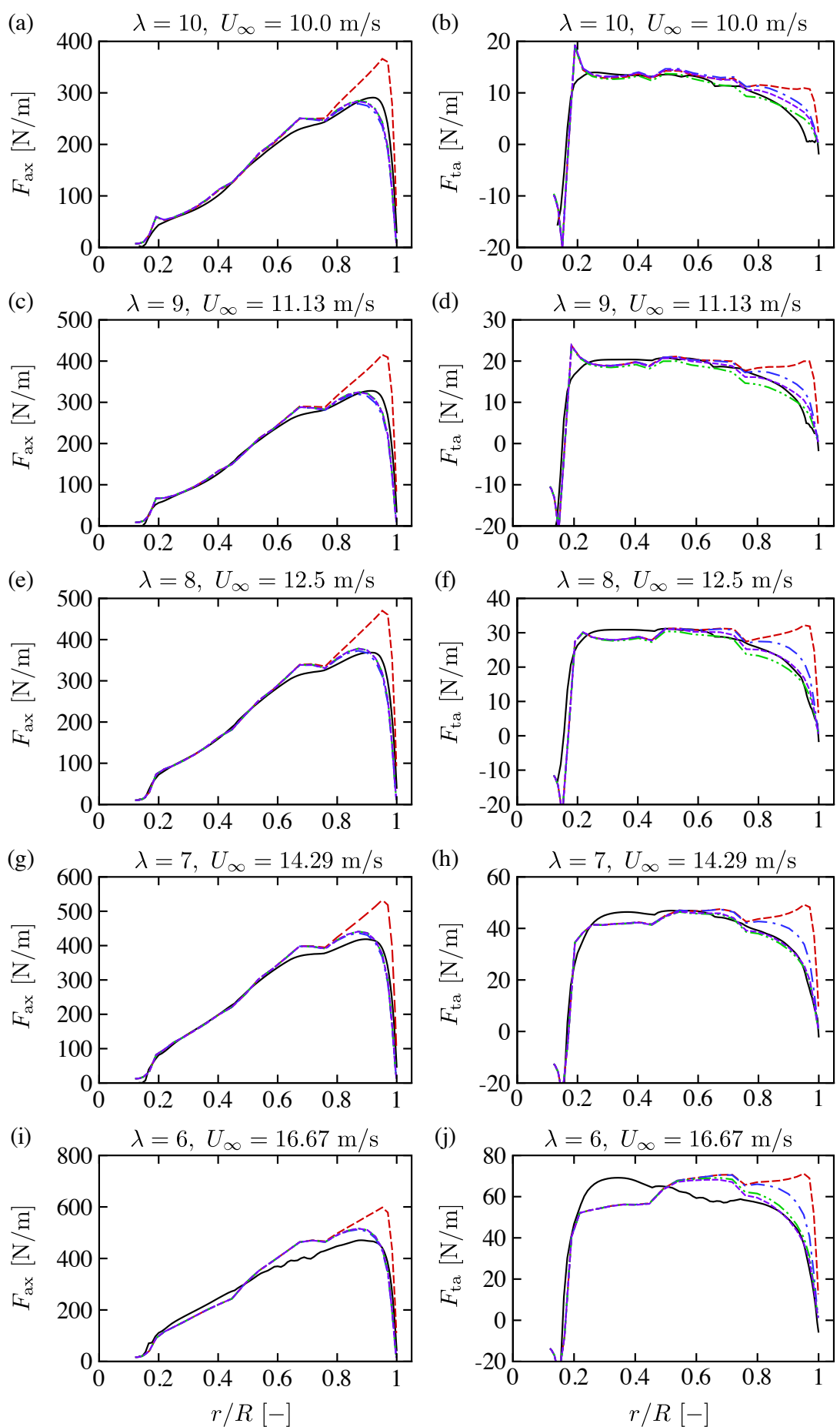

Blade Resolved

-.--- Actuator Line (Shen et al. Correction, Original Coefficients)

Actuator Line (No Correction)

Actuator Line (Shen et al. Correction, Curve Fit 1)

Actuator Line (Shen et al. Correction, Curve Fit 2)

Figure 8. Axial and tangential blade forces per unit span for tip speed ratios of 10, 9, 8, 7 and 6 , using the actuator line and blade resolved methods.

$0.007(1.6 \%)$ by curve fit 1 and $0.012(2.7 \%)$ by curve fit 2 . Furthermore, curve fit 1 achieved the closest overall agreement 

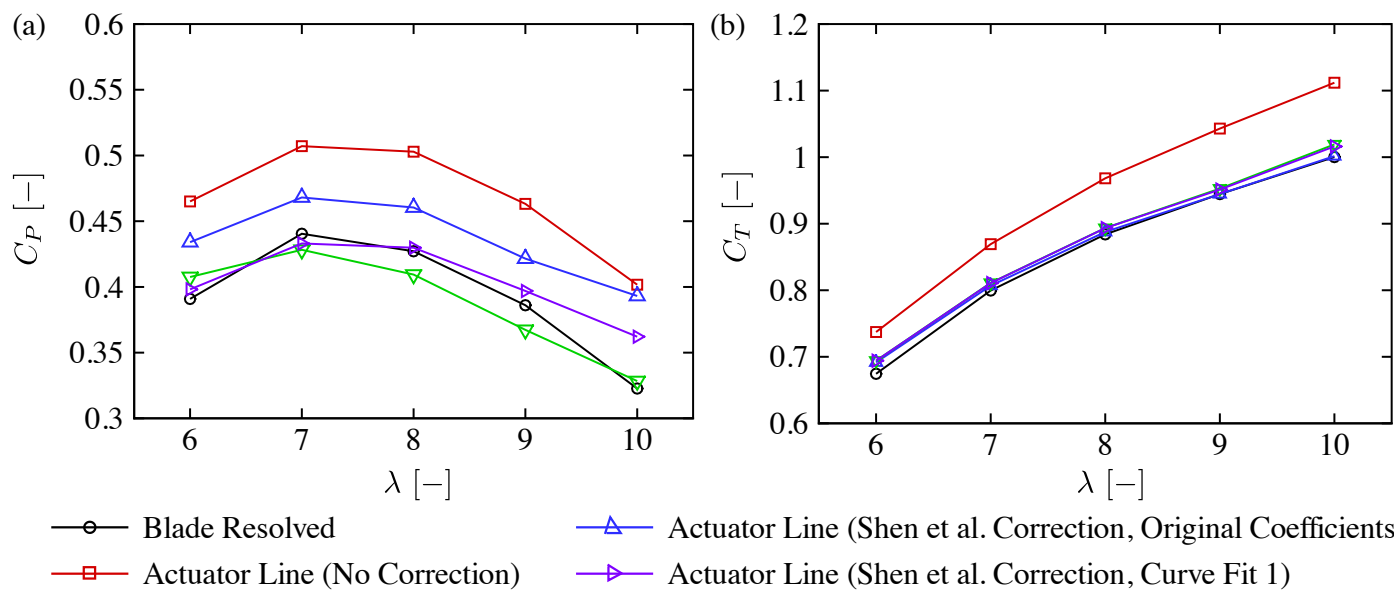

$\triangle$ Actuator Line (Shen et al. Correction, Original Coefficients)

$\rightarrow$ Actuator Line (Shen et al. Correction, Curve Fit 1)

$\checkmark$ Actuator Line (Shen et al. Correction, Curve Fit 2)

Figure 9. Thrust and power coefficients for the MEXICO rotor, computed using blade resolved and actuator line approaches.

with the blade resolved computations, as the curve fit for $g_{1, \text { ta }}$ was specific to the MEXICO rotor and did not include data points for the NREL Phase VI rotor or the Swedish WG 500 rotor.

At high tip speed ratios, the power coefficient computed with the corrected actuator line computations began to show a less comprehensive agreement with the blade resolved computations. By observing the tangential force per unit span in Fig. 8, this is seen to be due to the functional form of $F_{1}$ being insufficient to capture the spanwise variation near the blade tip. Furthermore, as the tangential force per unit span computed with the blade resolved computations tended to zero before the tip and the actuator line computations did not, the actuator line computations all over-predicted the power coefficient in Fig. 9. As discussed in section 5, $F_{1}$ was found to be less appropriate for the MEXICO rotor at high tip speed ratios and hence an alternative form for $F_{1}$ may lead to further improvement here.

\section{CONCLUSIONS}

It is imperative to include three-dimensional flow corrections (tip corrections) when using low order rotor models that rely on the flow independence principle to compute the blade forces. These tip corrections aim to account for the effect of pressure equalisation and the accompanying spanwise pressure gradients on the outboard sections, by reducing the axial and tangential forces per unit span. In this investigation, the tip correction factor of Shen et al. [5] was assessed (with the original empirical coefficients) for the case of actuator line simulations of the MEXICO rotor, and showed a reasonable performance improvement over similar actuator line computations with no correction applied. Further improvement was achieved by calibrating the correction factor specifically to the MEXICO rotor, which was achieved using data from blade resolved simulations.

An additional calibration was proposed for the correction factor using blade resolved data for the MEXICO rotor, along with existing data for the NREL Phase VI rotor and the Swedish WG 500 rotor. This approach also led to improved prediction of the axial and tangential forces per unit span when compared to the predictions using the original empirical coefficients proposed by Shen et al. [5], although this agreement was not as comprehensive as that achieved by the correction factor that was only calibrated to the computed MEXICO rotor data.

It was observed in the blade resolved computations of the MEXICO rotor that the tip loss (progressive unloading of the blade as the tip is approached) is more aggressive in the tangential direction than the axial direction. Whilst the original model coefficients proposed by Shen et al. [5] $\left(c_{1}=0.125, c_{2}=21.0\right)$ were found to be accurate for prediction of the unloading of axial force (and thus the rotor thrust), they provided insufficient tip loss when applied in the tangential direction. This lead to an over-prediction of the power delivered by the rotor $(6.39 \%$ at the design tip speed ratio). The newly calibrated coefficients for the tangential tip loss model $\left(c_{1}=0.1652, c_{2}=17.732\right)$ gave greater reduction in the outboard loading, leading to better prediction of the rotor power (a 1.6\% under prediction at the design tip speed ratio). In addition, a further minor improvement to the axial tip loss model could also be achieved using newly calibrated coefficients $\left(c_{1}=0.1215, c_{2}=21.39\right)$. The results presented in this investigation therefore emphasise that particular care should be taken when selecting appropriate empirical coefficients for horizontal axis rotor computations using reduced order methods. 


\section{ACKNOWLEDGEMENT}

The authors would like to thank EPSRC and Uniper for funding the CASE-Studentship for this project and the Advanced Research Computing facility at the University of Oxford where the computations where performed. The authors would also like to thank the consortium that carried out the EU FP5 project MEXICO. They provided a computer aided design (CAD) file containing the geometry of the MEXICO rotor blade that was used to generate the blade resolved mesh.

\section{REFERENCES}

1. Betz A. Schraubenpropeller mit geringstem Energieverlust. Mit einem Zusatz von L. Prandtl. Nachrichten von der Gesellschaft der Wissenschaften zu Gottingen, Mathematisch-Physikalische Klasse 1919; :193-217.

2. Goldstein S. On the vortex theory of screw propellers. Proc. R. Soc. A 1929; 123(792):440-465.

3. Burton T, Jenkins N, Sharpe D, Bossanyi E. Wind Energy Handbook. John Wiley \& Sons Ltd.: West Sussex, UK, 2011.

4. Glauert H. Aerodynamic Theory, chap. Airplane Propellers. Julius Springer: Berlin, 1935; 169-360.

5. Shen WZ, Mikkelsen R, Sørenson JN, Bak C. Tip loss corrections for wind turbine computations. Wind Energy 2005; 8:457-475.

6. Sørenson NN, Michelsen JA, Schreck J. Navier-Stokes predictions of the NREL Phase VI rotor in the NASA-Ames $80 \mathrm{ft}$ x $120 \mathrm{ft}$ wind tunnel. Wind Energy 2002; 5:151-169.

7. Shen WZ, Zhu WJ, Sørenson JN. Study of tip loss corrections using CFD rotor computations. Journal of Physics: Conference Series 2014; 555:012 094.

8. Wimshurst A, Willden RHJ. Extracting lift and drag polars from blade resolved computational fluid dynamics for use in actuator line modelling of horizontal axis turbines. Wind Energy 2016; :Accepted.

9. Lindenburg C. Investigation into rotor blade aerodynamics. Technical Report ECN-C-03-025, Energy Research Centre of the Netherlands (ECN) 2003.

10. Sant T. Improving BEM-based aerodynamics models in wind turbine design codes. PhD Thesis, DU Wind - Delft University Wind Energy Research Institute 2007.

11. Schepers JG, Boorsma K, Cho T, Gomez-Iradi S, Schaffarczyk P, Jeromin A, Shen WZ, Lutz T, Meister K, Stoevesandt B, et al.. Final report of IEA task 29, Mexnext (phase 1): Analysis of MEXICO wind tunnel measurements. Technical Report ECN-E12-004, Energy Research Centre of the Netherlands (ECN) February 2012.

12. Shen WZ, Zhu WJ, Sørenson JN. Actuator line/Navier-Stokes computations for the MEXICO rotor: comparison with detailed measurements. Wind Energy 2012; 15:811-825.

13. Wimshurst A, Willden RHJ. Validation of an actuator line method for tidal turbine rotors. The 26th International Ocean and Polar Engineering Conference, ISOPE: Rhodes (Rodos), Greece, 2016; TPC-2016-0825.

14. Sørenson JN, Shen WZ. Numerical modelling of wind turbine wakes. Journal of Fluids Engineering 2002; 124(2):393-399.

15. Schluntz J, Willden R. An actuator line based method with novel blade flow field coupling based on potential flow equivalence. Wind Energy 2015; 18(8):1469 - 1485.

16. Boorsma K, Schepers JG. Description of experimental setup, MEXICO measurements. Technical Report ECN-X-09OXX, Energy Research Centre of the Netherlands (ECN) 2009.

17. Patankar SV. Numerical Heat Transfer and Fluid Flow. Hemisphere Publishing Corporation: Washington DC (USA), 1980.

18. Sweby PK. High resolution schemes using flux limiters for hyperbolic conservation laws. SIAM Journal on Numerical Analysis 1984; 21:995-1011.

19. Luo JY, Issa RI, Gosman AD. Prediction of impeller-induced flows in mixing vessels using multiple frames of reference. IChemE Symposium Series, 136, 1994; 549-556.

20. Farrell PE, Maddison JR. Conservative interpolation between volume meshes by local galerkin projection. Comput. Methods Appl. Mech. Engrg. 2011; 200:89-100.

21. Bechmann A, Sørenson N, Zahle F. CFD simulations of the MEXICO rotor. Wind Energy 2011; 14:677-689. 